\title{
PROFONDEUR DE PENETRATION ET FORMATION DES RAIES DANS UNE ATMOSPHERE DIFFUSANTE
}

\author{
Y. FOUQUART \\ Laboratoire d'Optique Atmosphérique, Université de Sciences \\ et Techniques de Lille, France
}

\begin{abstract}
Résumé. L'étude systématique de la fonction de distribution du chemin optique des photons diffusés par une atmosphère diffusante finie permet de connaître la profondeur de pénétration du rayonnement dans un nuage; on peut alors obtenir une information précise sur l'épaisseur optique de la couche nuageuse dans laquelle a été formée une raie spectrale. La relation qui existe entre la fréquence de la raie observée et l'altitude de la région de l'atmosphère correspondante est ainsi précisément exprimée. La méthode qui est présentée ici, ainsi que quelques résultats préliminaires de Vénus, Jupiter ou Saturne permettent d'améliorer la connaissance de la structure interne des nuages.

L'application de cette méthode à l'interprétation des raies formées sur Vénus a permis de préciser que l'épaisseur optique des nuages, dans le cas du modèle de Hansen serait de l'ordre de 40 et leur sommet situé vers $60 \mathrm{~km}$ d'altitude.
\end{abstract}

\begin{abstract}
The systematic study of the distribution of the photon optical path in a finite scattering atmosphere gives the penetration depth of the radiation in a cloud; one can get then an accurate knowledge about the layer thickness in which the spectral line has been formed. The relation between the observed line frequency and the corresponding altitude is thus precisely expressed. The method is presented here with some preliminary results, it could be applied to the Venus, Jupiter and Saturne atmospheres to improve the knowledge of their clouds internal structure.

Using this method in case of Venus spectral lines it has been possible to deduce that for the Hansen's model, the optical depth of Venus clouds should be of the order of 40 and the cloud top closed to $70 \mathrm{~km}$ height.
\end{abstract}

\section{Introduction}

Ces dernières années de nombreux travaux ont été consacrés à l'étude de la formation des raies spectrales en milieu diffusant, citons ceux de Chamberlain (1965), Belton (1968), Sagan et Regas (1970), et Fouquart et Lenoble (1973). Ces travaux ont permis de rendre compte de l'effet de phase c'est-à-dire de l'augmentation de la largeur équivalente lorsque diminue l'angle de phase et de justifier l'existence des régimes limites. Cependant l'une des questions essentielles qui restent posées est celle du niveau de formation de la raie observée; en effet, dans le cas d'une atmosphère claire ce niveau peut être précisément défini mais dans le cas d'une atmosphère nuageuse l'impossibilité de déterminer le chemin suivi par les photons a conduit à considérer la pression et la température obtenues comme celles qui règnent au sommet des nuages, si ceux-ci sont très denses c'est certainement une bonne approximation mais si le libre parcours moyen des photons est grand c'est très certainement faux. La méthode que nous proposons ici permet de déterminer la pénétration du rayonnement observé, on peut ainsi définir le niveau de formation de la raie et quelques résultats intéressants concernant la couche nuageuse peuvent être déduits. 


\section{La distribution du chemin optique}

Dans ce but nous utilisons la distribution du chemin optique des photons diffusés. Considérons une couche plane parallèle d'épaisseur optique $\tau, \sigma$ est le coefficient de diffusion, $k_{c}$ le coefficient d'absorption du continu, $k_{v}$ le coefficient d'absorption du gaz à la fréquence $v$.

L'albédo de diffusion du continu est défini par la relation (1)

$$
\omega_{c}=\frac{\sigma}{\sigma+k_{c}}
$$

et l'albédo de diffusion à la fréquence $v$ par la relation (2)

$$
\omega_{v}=\frac{\sigma}{\sigma+k_{c}+k_{v}} .
$$

Dans ces conditions, et pour une direction d'incidence $\left(\mu_{0}, \varphi_{0}\right)$ et d'émergence $(\mu, \varphi)$, la distribution $p(\lambda)$ des photons ayant parcouru dans le continu un chemin optique $\lambda$ est défini par la relation (3)

$$
I\left(\omega_{v}\right)=I\left(\omega_{c}\right) \int_{0}^{\infty} p(\hat{\lambda}) e^{-r \lambda} \mathrm{d} \lambda,
$$

où $r$ est défini par la relation (4)

$$
r=\frac{k_{v}}{\sigma+k_{c}} \text {. }
$$

La fonction $p(\lambda)$ est donc la transformée de Laplace inverse de la fonction $I(r) / I\left(\omega_{c}\right)$, cette dernière fonction est interpolée au moyen des approximants de Pade (relation(6)).

$$
\frac{I(r)}{I\left(\omega_{c}\right)}=\frac{\sum_{i=1}^{N-1} b_{i} r^{i}}{\sum_{j=1}^{N} c_{j} r^{j}}
$$

On obtient alors $p(\lambda)$ sous la forme d'une série d'exponentielles (relation (7)).

$$
p(\lambda)=\sum_{m=1}^{N} A_{m} e^{\gamma_{m} \lambda}
$$

les $\gamma_{m}$ étant les racines du dénominateur et les $A_{m}$ les coefficients de la décomposition en éléments simples. La figure 1 représente les fonctions de distribution correspondant à diverses épaisseurs optiques pour les directions $\mu=\mu_{0}=1$. Il est clair que si un photon a pénétré jusqu'à une profondeur optique $\tau$ il a parcouru un chemin optique au moins égal à deux fois cette profondeur, dans ces conditions si $p_{\tau}(\lambda)$ est normalisée suivant la relation $(8)$

$$
\int_{0}^{\infty} p_{\tau}(\lambda) \mathrm{d} \lambda=\frac{I\left(\omega_{c}, \tau\right)}{I\left(\omega_{c}, \infty\right)}
$$




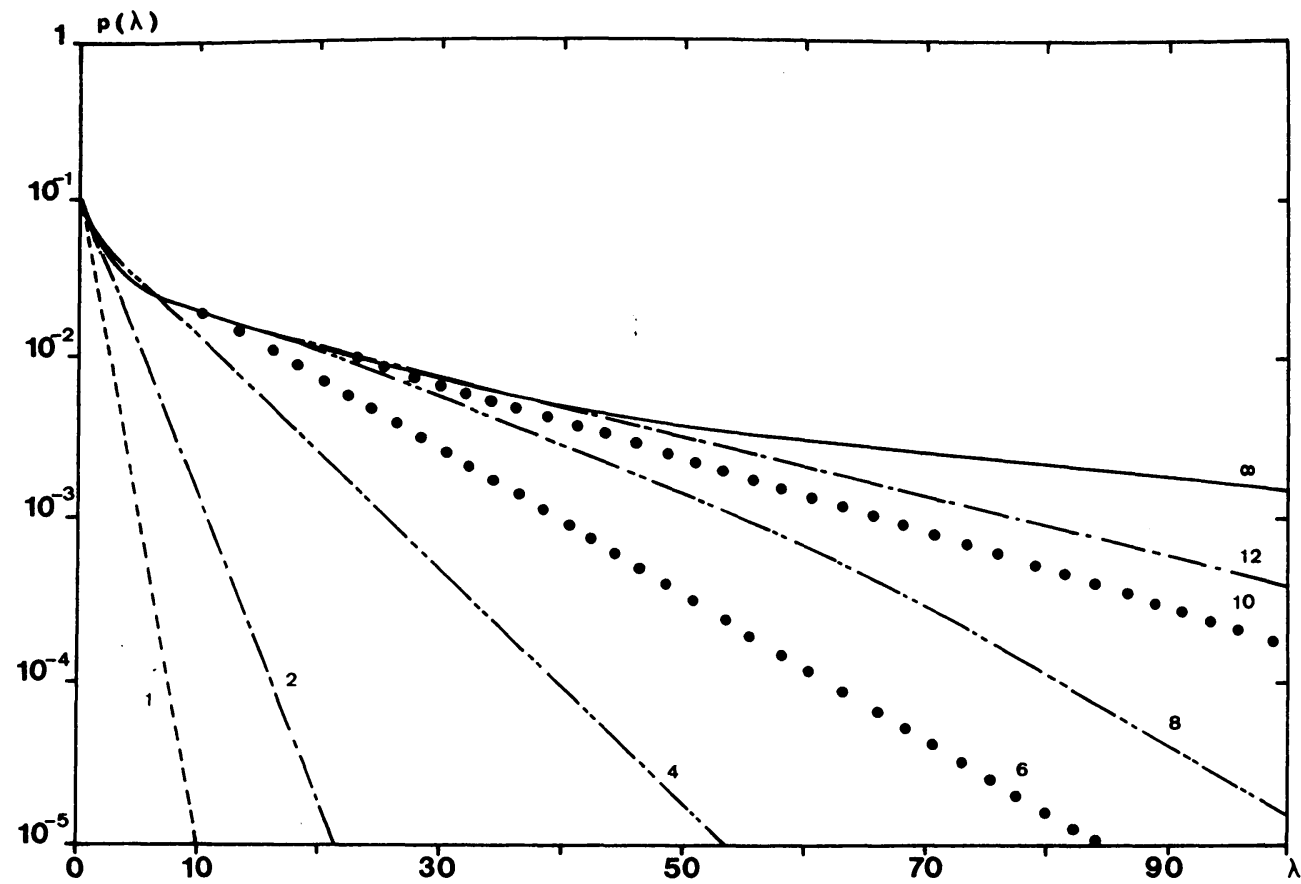

Fig. 1. Fonctions de distribution $p(\lambda)$ des photons correspondant à diverses épaisseurs optiques pour les directions $\mu=\mu_{0}=1$.

on aura:

$$
p_{\tau}(\lambda) \equiv p_{\infty}(\lambda) \quad \text { si } \quad \lambda \leqslant 2 \tau
$$

La connaissance des distributions correspondant à diverses épaisseurs optiques permet d'obtenir la probabilité de sortie d'un photon ayant parcouru au total un chemin optique $\lambda$ et pénétré jusqu'à une profondeur optique donnée. A titre d'exemple le tableau I présente, pour un chemin optique égal à 40 , le pourcentage de

\section{TABLEAU I}

Pourcentage de photons provenant de couches d'épaisseurs optiques $\tau$

\begin{tabular}{lrrrrrl}
\hline$\tau \leqslant$ & 4 & 6 & 8 & 10 & 12 & $\infty$ \\
\hline$\%$ & 2 & 20 & 57 & 76 & 94 & 100
\end{tabular}

photons provenant de couches de diverses épaisseurs optiques. On notera que dans ce cas $60 \%$ des photons ont pénétré jusqu'à une profondeur optique maximum comprise entre 6 et 10 . On peut donc définir le chemin optique moyen correspondant à une pénétration jusqu'à une profondeur optique comprise entre deux épaisseurs optiques consécutives $\tau_{1}$ et $\tau_{2}$ (relation 10 ). 


$$
\left\langle\lambda_{12}\right\rangle=\frac{I\left(\omega_{c}, \infty\right)}{I\left(\omega_{c}, \tau_{2}\right)-I\left(\omega_{c}, \tau_{1}\right)} \int_{0}^{\infty} \lambda\left(p_{\tau_{2}}(\lambda)-p_{\tau_{1}}(\lambda)\right) \mathrm{d} \lambda .
$$

\section{Etude des raies formées en atmosphère diffusante}

Pour une raie formée au cours de la réflexion du rayonnement dans une atmosphère diffusante, la largeur équivalente s'exprime par la relation (11)

$$
W=\int_{0}^{\infty} p(\lambda) W(\lambda b) \mathrm{d} \lambda,
$$

où $W(\lambda b)$ est la largeur équivalente d'une raie formée par absorption le long d'un trajet géométrique $L=\lambda /\left(\sigma+k_{\tau}\right)$ et $b$ est défini par (12)

$$
b=\frac{S M \omega_{c}}{2 \pi \alpha_{0} p}
$$

$S$ - est l'intensité de la raie,

$\alpha_{0}$-sa demi largeur,

$p$ - la pression,

$M$ - la 'quantité spécifique' c'est-à-dire le nombre de cm-atm de gaz absorbant contenu dans une colonne de longueur $1 / \sigma$.

Compte tenu de l'expression de la distribution $p(\lambda)$, pour une raie de Lorentz, $W$ s'exprime sous la forme (13):

$$
W=2 \pi \alpha_{0} p \sum_{m=1}^{N}\left(-\gamma_{m}\right)^{3 / 2}\left(2 b-\gamma_{m}\right)^{-1 / 2}
$$

en régime faible (b petit) on obtient l'expression (14)

$$
W \simeq S M \omega_{c} \sum_{m=1}^{N} \frac{A_{m}}{\left(-\gamma_{m}\right)^{2}}=S M \omega_{c}\langle\lambda\rangle
$$

et en régime fort l'expression (15)

$$
W \simeq\left(\pi \alpha_{0} p M S \omega_{c}\right)^{1 / 2} \sum_{m=1}^{N} \frac{A_{m}}{\left(-\gamma_{m}\right)^{3 / 2}}=2\left(\alpha_{0} p M S \omega_{c} \lambda\right)^{1 / 2}
$$

où $\langle\lambda\rangle$ est le chemin optique moyen défini par (16)

$$
\langle\lambda\rangle=\int_{0}^{\infty} \hat{\lambda} p(\hat{\lambda}) \mathrm{d} \lambda
$$


et $\bar{\lambda}$ est le chemin optique efficace défini par (17)

$$
\lambda^{1 / 2}=\frac{1}{2} \int_{0}^{\infty} \lambda^{1 / 2} p(\lambda) \mathrm{d} \lambda .
$$

\section{Application au cas de Vénus}

Cette méthode a été appliquée au cas des raies formées sur Vénus aux environs de $8000 \AA$. On a donc considéré un nuage de particules sphériques d'indice $n=1,46$ correspondant aux résultats de Hansen et Arking (1971), de granulométrie de type $C_{1}$ de Deirmendjann (1969), de rayon critique 0,8 $\mu$ avec un albedo continu de 0,999 en bon accord avec les valeurs d'albédo sphérique trouvées par Irvine (1968). Les calculs ont été effectués par la méthode des harmoniques sphériques avec la véritable fonction de phase déduite de la théorie de Mie.

On a tout d'abord calculé, pour un milieu d'épaisseur optique infinie les chemins optiques moyens et efficaces correspondant aux divers angles de phase et présentés au tableau II.

Les maximum situés vers $45^{\circ}$ correspondent à la pointe arrière de la fonction de phase qui est assez forte et donne une diffusion primaire plus importante au voisinage de $0^{\circ}$ diminuant ainsi les chemins optiques moyens correspondant.

Compte tenu des expressions des largeurs équivalentes des raies faibles et des raies fortes, il ressort clairement de ce tableau que:

(a) les raies faibles correspondant à des chemins optiques plus grands correspondent aussi à des pénétrations plus grandes que les raies fortes.

TABLEAU II

Chemins optiques moyens et

efficaces correspondant aux divers angles de phase, calculé pour: $n=1,46$, $\tau=\infty, r_{c}=0,8 \mu, \lambda=0,8 \mu$,

\begin{tabular}{rcr}
\multicolumn{3}{c}{$\omega_{c}=0,999$} \\
\cline { 1 - 2 } & \multicolumn{1}{c}{$\langle\lambda\rangle$} & \multicolumn{1}{c}{$\lambda$} \\
168 & 1,97 & 1,24 \\
145 & 13,35 & 4,89 \\
120 & 37,15 & 15,59 \\
106 & 51,65 & 23,85 \\
91 & 66,49 & 33,11 \\
74 & 77,57 & 41,54 \\
52 & 85,55 & 47,58 \\
45 & 89,53 & 48,25 \\
36 & 87,78 & 48,04 \\
25 & 81,00 & 44,36 \\
0 & 81,74 & 41,77
\end{tabular}


(b) l'effet de phase, pour une atmosphère diffusante homogène, produit d'abord une augmentation de la largeur équivalente jusque vers $45^{\circ}$ puis une diminution.

(c) cet effet de phase est plus important pour les raies faibles que pour les raies fortes.

On a ensuite calculé les chemins optiques moyens correspondant à une pénétration comprise entre deux épaisseurs consécutives et les résultats sont présentés au tableau

TABLEAU III

Chemins optiques moyens $\left\langle\lambda_{12}\right\rangle$ pour deux épaisseurs consécutives et différents angles de phase

\begin{tabular}{|c|c|c|c|c|c|c|c|c|}
\hline \multirow[t]{2}{*}{$\tau_{1}$} & \multirow[t]{2}{*}{$\tau_{2}$} & \multicolumn{7}{|c|}{ Angles de phase } \\
\hline & & 168 & 145 & 120 & 91 & 52 & 36 & 0 \\
\hline 0 & 0,25 & 1,32 & 0,94 & 0,70 & 0,70 & 0,60 & 0,27 & 0,26 \\
\hline 0,25 & 0,5 & 6,18 & 2,96 & 2,09 & 1,87 & 1,49 & 1,17 & 0,86 \\
\hline 0,5 & 1 & 8,81 & 5,49 & 4,06 & 3,55 & 2,88 & 2,40 & 1,90 \\
\hline 1 & 2 & 11,8 & 10,0 & 7,9 & 6,8 & 5,8 & 5,3 & 4,9 \\
\hline 2 & 4 & 19,3 & 18,0 & 15,6 & 13,8 & 11,7 & 11,6 & 10,8 \\
\hline 4 & 6 & & & 28,6 & 23,6 & 22,8 & 22,2 & 21,6 \\
\hline 6 & 8 & & & & & 35,9 & 35,4 & 31,7 \\
\hline 8 & 10 & & & 47 & & 56,9 & 52,6 & 51,9 \\
\hline 10 & 12 & & & & 108 & 69 & 66 & 67 \\
\hline
\end{tabular}

III pour différents angles de phase. De ces résultats on peut déduire le niveau moyen de formation des raies faibles et des raies fortes par comparaison des tableaux II et III. Puisqu'il s'agit là d'une information statistique moyenne on pourra considérer que la pression efficace de formation des raies est la pression qui règne à leur niveau moyen de formation.

Bien que ce tableau ne soit pas très détaillé, on peut déjà remarquer que la profondeur optique de formation des raies est sensiblement identique entre 0 et $52^{\circ}$, de l'ordre de 8 pour les raies fortes et de l'ordre de 12-13 pour les raies faibles.

Si l'on admet que les raies observées par Gray-Young (1970) aux environs de $8000 \AA$ sont des raies fortes, on peut déduire la pression et le produit $M_{p}$ de la 'quantité spécifique' par la pression à partir de l'expression des largeurs équivalentes de raies fortes, on obtient alors $M_{p} \simeq 0,03 \mathrm{~km}$ atm $^{2}$ et $p$ de l'ordre de $200 \mathrm{mb}$, résultats déjà obtenus* de $1 \mu$. Dans ces conditions il est possible de déterminer l'altitude du sommet des nuages et le libre parcours moyen des photons. Nous avons, pour cela, utilisé le modèle d'atmosphère de Marov (1972) et les résultats obtenus en supposant les raies formées à diverses altitudes sont présentés au tableau IV. Les mesures de flux effectuées par la sonde Venera VIII ont montré que la limite inférieure des nuages était située vers $35 \mathrm{~km}$ et qu'il y subsistait environ $1 \%$ du flux solaire incident, ce qui correspondrait d'après Herman et al. (1974) à une épaisseur optique de l'ordre de la centaine dans le

* Par L. Gray et Belton mais aux environs. 
TABLEAU IV

Altitude du sommet des nuages d'après le modèle de Marov

\begin{tabular}{lllllll}
\hline$Z(\mathrm{~km})$ & $T(\mathrm{~K})$ & $p(\mathrm{~atm})$ & $n\left(\mathrm{~m}^{-3}\right)$ & $n / N_{0}(\mathrm{~atm})$ & $\sigma+\mathrm{k}_{c}\left(\mathrm{~km}^{-1}\right)$ & $\begin{array}{l}\text { Altitude du } \\
\text { sommet }(\mathrm{km})\end{array}$ \\
\hline 56 & 285 & 0,485 & $1,2 \times 10^{25}$ & 0,448 & 7,244 & 57,1 \\
58 & 271 & 0,340 & $9,1 \times 10^{24}$ & 0,350 & 3,960 & 60 \\
60 & 260 & 0,250 & 6,8 & 0,254 & 2,116 & 63,8 \\
62 & 250 & 0,178 & 5,0 & 0,187 & 1,107 & 69,2 \\
64 & 240 & 0,124 & 3,6 & 0,134 & 0,556 & 78,5 \\
66 & 237 & 0,085 & 2,5 & 0,094 & 0,265 & 96,2 \\
68 & 234 & 0,059 & 1,7 & 0,0635 & 0,125 & 132 \\
70 & 231 & 0,040 & 1,2 & 0,0448 & 0,062 & 203 \\
\hline
\end{tabular}

visible. Dans ces conditions, le meilleur accord semble être obtenu en fixant le niveau de formation des raies vers 60 à $62 \mathrm{~km}$. Compte tenu des températures de rotation observées, voisines de $240 \mathrm{~km}$ et de la pression obtenue, nous avons fixé le niveau de formation à $62 \mathrm{~km}$, ce qui donne un libre parcours moyen de $1,1 \mathrm{~km}$ et fixe le sommet des nuages vers $70 \mathrm{~km}$ puisque la profondeur optique de formation est d'environ 8 . Dans ces conditions, la pression efficace de formation est de $178 \mathrm{mb}$ et l'épaisseur optique des nuages de l'ordre de 40 . Cette dernière valeur est sans doute trop faible mais la valeur du produit Mp est inversement proportionnelle au chemin optique

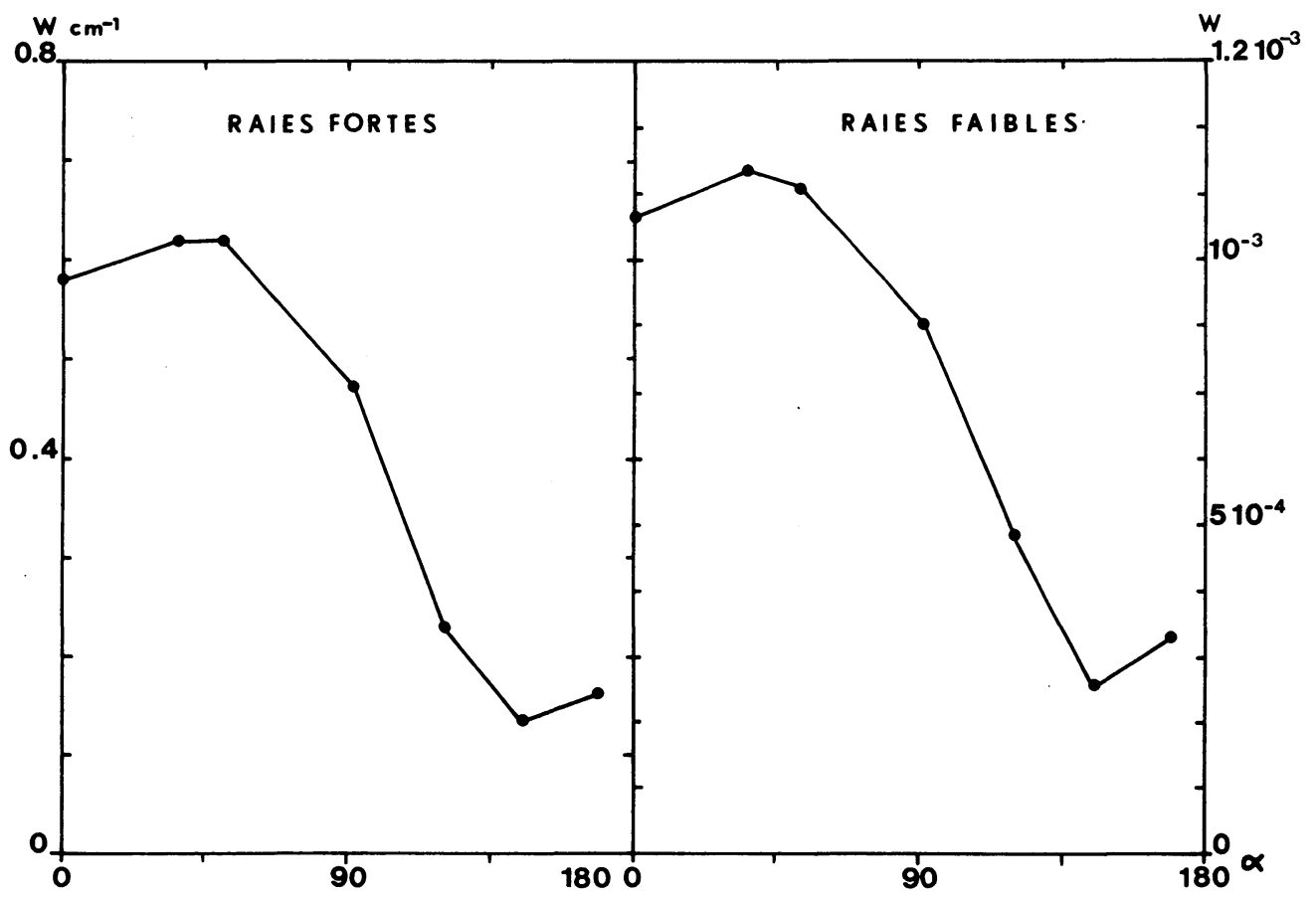

Fig. 2. Courbes de phase de la largeur équivalente tracées pour une raie d'intensité $0,67 \mathrm{~km}^{-1}$ $(\mathrm{cm}-\mathrm{atm})^{-1}$ et une raie faible d'intensité 1000 fois plus petite. 
efficace qui augmente lorsque l'albédo continu augmente; en choisissant pour $\omega_{c}$ la valeur 0,9999 le chemin optique efficace serait de 94 et le produit Mp serait voisin de 0,015 , le libre parcours moyen serait alors de 4 à $500 \mathrm{~m}$ et l'épaisseur optique de nuage de l'ordre de 80 . La profondeur optique de formation n'a pas été calculée, faute de temps, mais sa variation ne serait pas considérable et compte tenu du fait que le libre parcours moyen serait divisé par 2, l'altitude du sommet des nuages ne varierait pas sensiblement. Cependant cette dernière valeur de l'albédo continu est sans doute un peu trop grande si l'on s'en réfère aux valeur d'albédo sphérique, il serait sans doute préférable de choisir une valeur voisine de 0,9995, mais étant donné la précision des diverses mesures effectuées, l'ordre de grandeur des résultats obtenus ici semble satisfaisant.

Les courbes de phase de la largeur équivalente ont été tracées dans le cas sélectionné (niveau de formation $62 \mathrm{~km}$, albédo continu 0,999) et sont présentées (figure 2) pour une raie d'intensité $0,67 \mathrm{~km}^{-1}(\mathrm{~cm}-\mathrm{atm})^{-1}$ et une raie faible d'intensité 1000 fois plus petite. La pression efficace de formation dans le nuage est donc $178 \mathrm{mb}$ pour la raie forte et $270 \mathrm{mb}$ pour la raie faible, la pression efficace au dessus du nuage, en atmosphère claire étant de $20 \mathrm{mb}$. On remarquera que l'allure des courbes respecte les observations de Gray-Young et al. (1971) sans qu'il soit nécessaire de faire intervenir un deuxième nuage ainsi qu'avait cru devoir le conclure Hunt (1972); l'effet de remontée de la courbe de phase étant essentiellement dû à la pointe arrière du diagramme de diffusion.

\section{Conclusion}

En conclusion, l'utilisation de la distribution du chemin optique des photons diffusés permet de fixer avec précision la profondeur moyenne de pénétration du rayonnement dans une couche diffusante, le niveau de formation des raies fortes ou des raies faibles peut alors être déterminé et relié à la pression efficace de formation. L'application de cette méthode au cas de Vénus a permis de déterminer approximativement l'altitude du sommet des nuages et le libre parcours moyen des photons et de rendre compte de l'effet de phase.

\section{Bibliographie}

Belton, M.: 1968, J. Atmospheric Sci. 25, 596.

Chamberlain, J. W.: 1965, Astrophys. J. 141, 1184.

Deirmendjann, D.: 1969, The Electromagnetic Scattering on Spherical Polydropersions, Elsevier, New York.

Fouquart, Y. et Lenoble, J.: 1973, J. Quant. Spectrosc. Radiat. Transfer 13, 447.

Gray-Young, L. D.: 1970, Icarus 13, 449.

Gray-Young, L. D., Schorn, R. A., Barker, E. S., et Woszczyk, A.: 1971, Acta Astron. $21,329$.

Hansen, J. E. et Arking, A.: 1971, Science 171, 669.

Herman, M., Devaux, C., et Deuze, J. L.: 1974, sous presse.

Hunt, G. E.: 1972, J. Quant. Spectrosc. Radiat. Transfer 12, 405.

Irvine, W. M.: 1968, J. Atmospheric Sci. 25, 610.

Marov, M. Ya.: 1972, Icarus 16, 415.

Sagan, C. et Regas, J. L.: 1970, Comments Astrophys. Space Phys. 2, 116; 138; 161. 